\title{
Reflexiones sobre los orígenes de la formación en Terapia Ocupacional en Argentina
}

\author{
Reflections on the origins of Occupational Therapy \\ training in Argentina
}

\author{
María Marcela Bottinelli ${ }^{1}$ \\ Mariela Nabergoi ${ }^{2}$ \\ Marcela Cecilia Mattei ${ }^{3}$ \\ Federico Juan Manuel Zorzoli ${ }^{4}$ \\ Francisco Manuel Díaz ${ }^{5}$ \\ Natalia Milagros Spallato ${ }^{6}$ \\ Maureen Mulholland ${ }^{7}$ \\ María Magdalena Martínez Del Pezzo Bredereke ${ }^{8}$ \\ Agustina Mariel García Sartirana ${ }^{9}$ \\ Julieta Briglia ${ }^{10}$ \\ Sara María Daneri ${ }^{11}$
}

Recibido: 14 de octubre 2016 • Aceptado: 5 de diciembre 2016

Botinelli, M., Nabergoi, M., Mattei, M., Zorzoli, F., Díaz, F., Spallato, N.,... Daneri, S. (2016). Reflexiones sobre los orígenes de la formación en Terapia Ocupacional en Argentina. Revista Ocupación Humana, 16 (2), 11-25.

Resumen: Esta investigación tuvo como finalidad caracterizar el surgimiento y desarrollo de la formación de terapeutas ocupacionales en Argentina entre 1956 y 1965, identificando acontecimientos relevantes ocurridos desde el inicio de la carrera en la Escuela Nacional

\footnotetext{
${ }^{1}$ Licenciada en Psicología. Especialista y magíster en Metodología de la Investigación Científica. Doctora en Salud Mental Comunitaria. Docente investigadora Universidad Nacional de San Martín, Universidad de Buenos Aires, Universidad Nacional de Lanús. Buenos Aires, Argentina.mmbottinelli@yahoo.com.ar

${ }^{2}$ Licenciada en Terapia Ocupacional. Especialista en Metodología de la Investigación Científica. Doctora en Salud Mental Comunitaria. Docente investigadora Universidad de Buenos Aires, Universidad Nacional de San Martín, Universidad Nacional de Lanús. Buenos Aires, Argentina. mnabergoi@yahoo.com.ar

${ }^{3}$ Licenciada en Terapia Ocupacional. Equipo de investigación Universidad Nacional de San Martín. Buenos Aires, Argentina. marcelamattei@hotmail.com

${ }^{4}$ Licenciado en Terapia Ocupacional. Maestrando en Salud Mental Comunitaria. Docente investigador Universidad Nacional de San Martín. Buenos Aires, Argentina.jmzorzoli@hotmail.com

${ }^{5}$ Licenciado en Audiovisión. Especialista en Metodología de la Investigación Científica. Docente investigador Universidad Nacional de Lanús. Buenos Aires, Argentina. franciscomdiaz@hotmail.com

${ }^{6}$ Licenciada en Terapia Ocupacional. Maestranda en Metodología de la Investigación Científica. Docente investigadora Universidad Nacional de San Martín. Buenos Aires, Argentina. nataliaspallato@hotmail.com

${ }^{7}$ Licenciada en Terapia Ocupacional. Equipo de investigación Universidad Nacional de San Martín. Instituto Municipal de Rehabilitación de Vicente López, Dr Anselmo Marini. Buenos Aires, Argentina. maureenmulvey@ hotmail.com

${ }^{8}$ Licenciada en Terapia Ocupacional. Equipo de investigación Universidad Nacional de San Martín. Buenos Aires, Argentina. magdabre@yahoo.com.ar

${ }^{9}$ Licenciada en Terapia Ocupacional. Docente investigadora Universidad Nacional de San Martín. Hospital de Rehabilitación Manuel Rocca. Buenos Aires, Argentina. toagustinagarcia@gmail.com

${ }^{10}$ Licenciada en Terapia Ocupacional. Equipo de investigación Universidad Nacional de San Martín. Centro de Salud y Acción Comunitaria 9.juliet 173@hotmail.com

${ }^{11}$ Licenciada en Terapia Ocupacionā. Maestranda en Ciencias Sociales y Humanidades, mención en Sociología. Equipo de investigación Universidad Nacional de San Martín. Docente Instituto Universitario Gran Rosario. Buenos Aires, Argentina.saramdaneri@gmail.com
} 
de Terapia Ocupacional - ENTO. Los datos fueron recolectados mediante entrevistas en profundidad y utilizando metodologías propias de la historia oral. Asimismo, se trabajó con fuentes primarias y secundarias como documentos de época, material de archivo y planes de estudio. El análisis permite ubicar a la ENTO en el marco de la apertura de carreras de las áreas de salud y ciencias sociales, el proceso de incorporación de las mujeres a la educación superior, y acciones de cooperación internacional para la instalación del modelo de rehabilitación y la formación en disciplinas afines dentro de una estrategia desarrollista. Con influencia de la WFOT, tomando el modelo de la Dorset House School, a través de acciones de cooperación y un sistema de becas se formaron profesionales que desarrollaron la disciplina en distintas regiones de Argentina y otros países de Latinoamérica. Estos resultados permiten potenciar los diálogos hacia la comprensión de los procesos comunes y distintivos que dieron lugar a la institucionalización de Terapia Ocupacional en nuestro continente.

Palabras clave: Terapia Ocupacional, rehabilitación, historia, recursos humanos en salud, educación profesional.

\begin{abstract}
The purpose of this investigation was to characterize the origin and development of the occupational therapy training in Argentina between 1956 and 1965, identifying relevant events that took place since the beginning of the Occupational Therapy program at the Escuela Nacional de Terapia Ocupacional (National School of Occupational Therapy). The data was collected through in-depth interviews and using methodology pertinent to oral history. Primary and secondary sources, such as documents pertaining to that period, archiving material and study plans were also used. The analysis allows to locate the ENTO within the framework of the opening of programs in social sciences and health areas, the process of incorporation of women into higher education, and the international cooperation actions for the installation of the rehabilitation model and for training in related disciplines within a developmental strategy. Professionals who developed the discipline in different regions of Argentina and other Latin American countries were formed with the influence of WFOT and the application of Dorset House School model. These results strengthen the dialogue towards the understanding of the common and distinctive processes that gave rise to the institutionalization of Occupational Therapy in our continent.
\end{abstract}

Key words: Occupational therapy, rehabilitation, history, human resources in health, professional education.

\section{Introducción}

Los campos profesionales son definidos por los actores sociales que en un contexto determinado los sustentan y los promueven, tanto a nivel local como internacional. Ellos están determinados por modelos explícitos e implícitos en los currículos o planes de formación que, en el caso de los procesos de inicio de una disciplina o de la fundación o apertura de una carrera, marcan de manera matricial las bases de las prácticas y los aportes que esta realizará a la sociedad, y permiten comprender los avatares de su desarrollo y potencialidad.

Asimismo, se pueden relevar períodos de estabilidad y desarrollo de las profesiones, campos teóricos y de prácticas, así como períodos de cambios, propuestas y virajes hacia nuevas necesidades. Toda profesión requiere estar abierta a la detección de dichos momentos, de modo que sea posible adaptar sus prácticas y conocimientos a las nuevas demandas y necesidades, 
y dar cuenta de todas las funciones del quehacer científico y profesional, a saber: la producción de conocimientos por los conocimientos mismos, la producción de conocimientos por las prácticas que de ellos se deriven y la producción de conocimientos para la retroalimentación social (Samaja, 2003; Bottinelli 2003).

En este sentido, la investigación parte de la premisa de que toda disciplina tiene una dimensión histórica que debe ser revisada y conocida para comprenderla en profundidad, tanto en el surgimiento de su campo de conocimientos y prácticas como en su posicionamiento actual. Ello permite pensar sus aportes y potencialidades a futuro, tanto al interior de la disciplina como en la formación de nuevos profesionales y en la identificación de las necesidades sociales que los convocan, para realizar aportes en los campos en los que participa (Bottinelli, 2003). Es por ello que el proceso de revalorización del pasado constituye una posición epistemológica, ética y teórica del equipo de investigación.

En este marco y considerando que el Instituto de Ciencias de la Rehabilitación y el Movimiento de la Universidad Nacional de San Martín (ICRMUNSaM) albergan a la primera entidad formadora de terapeutas ocupacionales en Argentina, y que la Escuela Nacional de Terapia Ocupacional (ENTO) es el lugar donde se formaron profesionales que abrieron los primeros servicios y escuelas de Terapia Ocupacional del país y en algunos países de Latinoamérica, nos propusimos caracterizar el surgimiento y desarrollo de la formación de terapeutas ocupacionales en Argentina en el período 19561965. Este período abarca, desde la creación de la Comisión Nacional de Rehabilitación del Lisiado, en el marco de la cual se inscribió la carrera, y la realización de los primeros cursos breves de formación por parte de la terapeuta ocupacional inglesa Elizabeth Hollings; pasa por la creación de la Escuela Nacional de Terapia Ocupacional (ENTO) en 1959, y va hasta el momento en que las egresadas argentinas se hicieron cargo de la dirección de la carrera y de la formación en materias específicas de la disciplina.

\section{Metodología}

Se trabajó con un diseño de tipo exploratorio descriptivo, retrospectivo, con metodologías predominantemente cualitativas, integrando diferentes técnicas de historia oral y de registro documental. El valor de estas técnicas reside en la posibilidad de captar los sentidos y los significados de los acontecimientos humanos desde la perspectiva de los propios actores. La revisión, análisis y triangulación de la información recolectada con material de archivo y bibliografía secundaria permitió someter las fuentes orales a la crítica documental (Valles, 2003) y poner en relación los discursos con sus condiciones históricas de producción, propios de un enfoque hermenéutico-dialéctico (De Souza Minayo, 2009).

En este sentido, se trabajó utilizando herramientas propias de la historia oral tales como relatos de vida (Bertaux, 1999), que fueron recolectados a través de entrevistas en profundidad 
con terapeutas ocupacionales y otros actores vinculados con la creación e inicios de la carrera. Se formularon preguntas estructuradas y no estructuradas, organizadas por ejes temáticos.

Para la selección de las terapistas ocupacionales y la realización de entrevistas se conformó una muestra intencional utilizando la técnica de bola de nieve. Los criterios de inclusión fueron: primeros alumnos, primeros profesionales, primeros supervisores de práctica clínica, fundadoras de asociaciones de profesionales, primeros docentes, primeras directoras de carreras.

Así mismo, se recolectaron documentos de época, material de archivo, planes de estudio y bibliografía secundaria. Varios de estos documentos fueron donados por las entrevistadas y se organizaron en la conformación de un archivo digital documental.

Con motivo de la investigación identificamos e iniciamos contactos con quienes pudieran brindarnos información a través de fuentes primarias: las primeras docentes del equipo inglés; donaciones y material del Dorset House Archive y la British Association of Occupational Therapists, así como también de colegas que viven en el exterior y de numerosas terapistas ocupacionales de nuestro país. Para el control de la información recolectada y la pertinencia de la interpretación de resultados se utilizaron las técnicas de triangulación de fuentes y vigilancia del cumplimiento de criterios de rigor para diseños cualitativos (Sandelowski, citado en Zaldúa et al, 2010). Para el criterio de auditabilidad se realizaron reuniones de discusión de resultados con diferentes actores. Dadas las características del diseño, el procesamiento, sistematización y análisis de la información se realizó de manera procesual y paralela a la profundización teórica y la recolección de datos. El análisis incluyó la revisión intra e interentrevista, triangulación de fuentes y contextualización dentro de las políticas de salud nacionales y las principales tendencias internacionales en Terapia Ocupacional y en rehabilitación.

Algunas de las líneas de análisis propuestas inicialmente incluyeron la identificación de momentos relevantes y su contextualización en los marcos de la historia de la rehabilitación, de la salud en el país y en el ámbito internacional (Isuani \& Mercer, 1988; Veronelli \& Veronelli, 1991; Belmartino \& Bloch, 1994; Belmartino, 2005). El procesamiento y análisis de documentos (fuentes secundarias) permitió comprender las dificultades que atravesaba Argentina para la época y los diferentes esfuerzos por crear la carrera en el país y en la región. Además, del proceso de entrevistas surgieron nuevos elementos y líneas de análisis a considerar, tales como los modelos implícitos y explícitos en los planes de estudio, los debates paradigmáticos de la época, la creación de nuevos espacios profesionales a través de programas de becas, las características de los actores involucrados y los requisitos de acceso a la formación y a cargos, entre otras.

\section{Resultados}

La revisión de bibliografía secundaria y de antecedentes permitió identifi- 
car en el ámbito nacional los productos de esfuerzos individuales y colectivos realizados desde la Asociación Argentina de Terapistas Ocupacionales (Monzón \& Risiga, 2004a, 2004b; Fortain, 2003), trabajos finales de grado y posgrado (Testa, 2011, 2012; Testa \& Spampinato, 2010; Daneri, 2005, 2010; Gracerón, 2000), algunos artículos, presentaciones en congresos y capítulos de libro que hacen referencia al tema (Sbriller \& Suter, 1985; Warschavski \& Sbriller, 1990; Sbriller, 1997; Paganizzi, 2007; Gomez Mengelberg, 2007a, 2007b; Demiryi, 2001; Destuet, 1999; Villarroel, 1985), así como otros trabajos individuales llevados a cabo por interés particular de estudiantes de grado y graduados. En estos documentos se refieren algunas dificultades respecto de la recuperación de la historia de la profesión en el país, incluyendo la falta de recursos para trabajar el tema en profundidad, la escasez de archivos institucionales que consultar y la fragmentación de la información, que llevan a la necesidad de reconstruir la historia a través de los relatos de quienes la vivieron.

Por su parte, en el ámbito latinoamericano se destacan los trabajos de Guajardo (2010) y Gómez Lillo (2012) en Chile, los de Teixeira Soares (1991) y Da Rocha Medeiros (2008) en Brasil, Forn (2007) en Venezuela y el de TrujiIlo (2002) en Colombia. En los últimos años además se han sumado nuevas incitativas con enfoques más participativos, como los de Fernández, García, Duarte \& Cruz (2016) en Colombia, y el del Grupo de Investigación Terapia Ocupacional: memórias, histórias e
Fundamentos, del Programa de PosGraduación en Terapia Ocupacional de la Universidade Federal de São Carlos (UFSCar) en Brasil, coordinado por Roseli Esquerdo Lopes y Ana Paula Malfitano. En otros países sobresalen los de Moruno y Talavera (2007), Peters (2006), Schwartz (2005), Wilcock (2002), Reed (1998), Kielhofner \& Burke (1977), Collins (1989), entre otros.

\section{Momentos relevantes y periodización}

Los orígenes de la formación en Terapia Ocupacional en Argentina se remontan a las epidemias de polio ocurridas entre los años 1953 y 1955, momento histórico que dio lugar al desarrollo de las disciplinas relacionadas con la rehabilitación en Latinoamérica y a la introducción en la región de los conocimientos y prácticas de salud desarrollados en los países del norte durante el período de posguerra mundial (Teixeira Soares, 1991; Sbriller, 1997; Guajardo, 2010).

Ese acontecimiento impulsó la introducción de la Terapia Ocupacional como profesión de salud en Argentina, mediante un convenio del Estado con el Gobierno británico; tal acuerdo se concretizó a través de la contratación de un grupo de terapistas ocupacionales inglesas, quienes en el año 1959 viajaron al país para organizar la formación profesional y dictar los cursos hasta que hubiera egresados en el país que pudieran hacerse cargo de la misma. La institucionalización de esta formación se materializó en la creación de la Escuela Nacional de Terapia Ocupa- 
cional (ENTO), en el marco de la Comisión Nacional de Rehabilitación del Lisiado, dependiente del Ministerio de Salud Pública (Spallato, 2010; Nabergoi et al, 2011).

Se pudieron diferenciar tres periodos dentro de la década estudiada: 1. los antecedentes de creación de la Escuela (1956-1959), que van desde la creación de la Comisión Nacional de Rehabilitación del Lisiado (CNRL) hasta la creación de la ENTO, e incluye los cursos de formación de auxiliares de Terapia Ocupacional por parte de Elizabeth Hollings en 1956 y Ruby Kroom en 1957 (Figura 1); 2. un primer período de la ENTO con el equipo de docentes inglesas a cargo de la Escuela (1959-1963), se distinguen en este período un primer grupo a cargo de Miss MacDonald y un segundo grupo con matices diferentes en cuanto a sus antecedentes y formación, y 3. el comienzo del proceso de "argentinización", iniciado con el traspaso de la gestión y las tareas docentes a las egresadas argentinas (1963-1965).

Los cursos de Terapia Ocupacional y Fisioterapia previos a la creación de la ENTO estuvieron a cargo del equipo inglés liderado por Mónica Martin Jones, con Elizabeth Hollings como docente de Terapia Ocupacional. Quienes participaron en estos cursos, como Carmen Forn, Marta Fortain y Helvia Llambí, fueron luego estudiantes de la primera cohorte de la ENTO y líderes de la Terapia Ocupacional en el país.

Figura 1. Primeras egresadas y docentes de los cursos de Terapia Ocupacional y Fisioterapia previos a la creación de la ENTO.

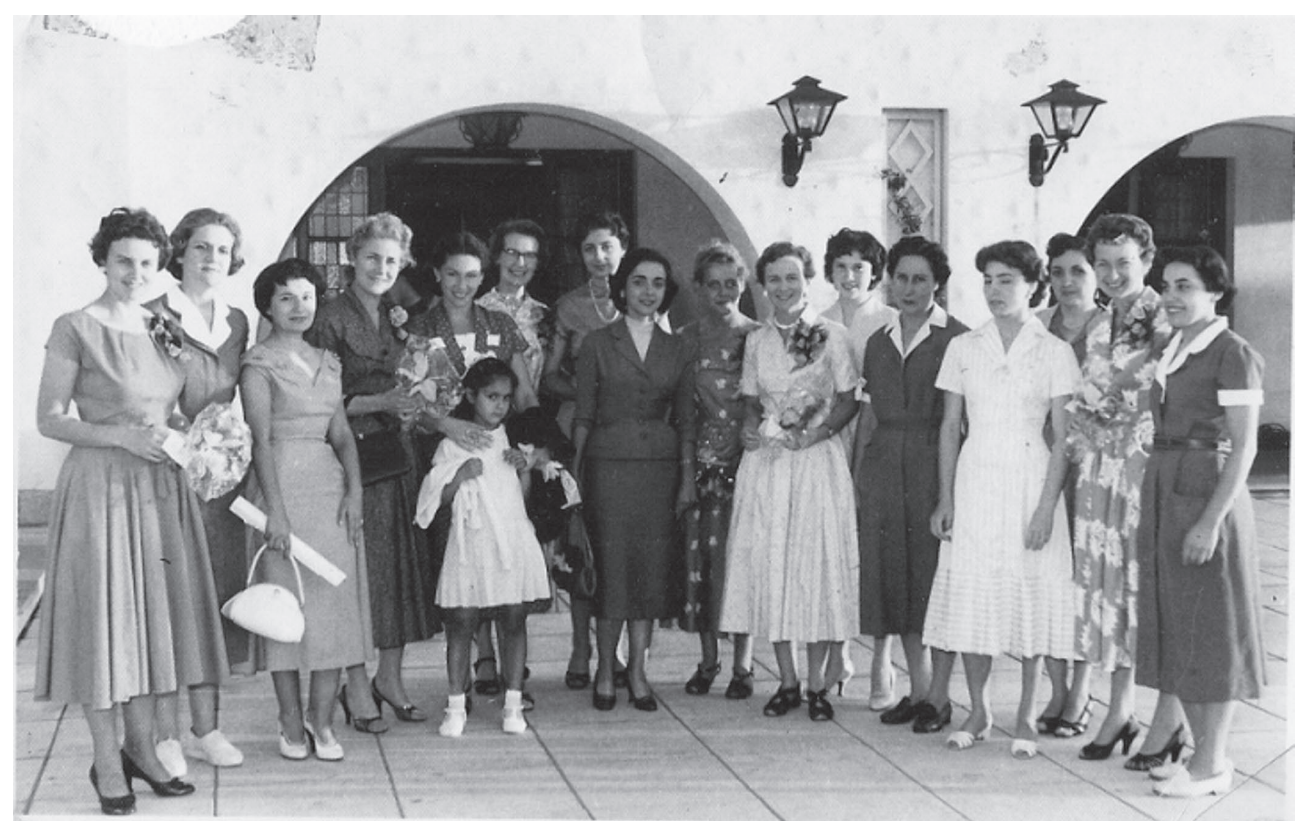

Foto: Archivo digital de las investigaciones llevadas a cabo por los autores. Año 1956 (probable). 


\section{Contextualización en los marcos de la historia de la rehabilitación y de la salud en Argentina y en el ámbito internacional}

Al poner en diálogo las narrativas con los diferentes contextos meso y macro políticos, ubicamos la ENTO en el marco de la apertura de diversas carreras de las áreas de la salud y las ciencias sociales (Ej. Psicología, Sociología, Ciencias de la Educación y Escuela de Salud Pública), así como de los cambios en el campo de la atención en discapacidad y en salud mental. Pudimos observar que si bien el emergente socio sanitario de la creación de la carrera fue la epidemia de poliomielitis, otras necesidades sociales y procesos de debate y transformación tenían lugar en ese momento, los cuales se articularon con la nueva formación y abrieron nuevas líneas de indagación no previstas en dicho proyecto.

Como parte de la estrategia desarrollista, característica del gobierno del entonces Presidente Frondizi, la creación de la ENTO también forma parte de un conjunto de acciones de cooperación internacional para la instalación del modelo de rehabilitación y formación en las disciplinas afines, promovidas por organismos internacionales como la Organización Mundial de la Salud (OMS), la Oficina Sanitaria Panamericana y la Organización Internacional del Trabajo (OIT). Otras acciones de cooperación que determinaron la formación y creación de la ENTO incluyeron acuerdos entre el Gobierno Argentino y el British Council. Todo ello implicó que la capacitación inicial en Terapia Ocupacional estuviese marcada por un enfoque de rehabili- tación importado centralmente desde Inglaterra, a diferencia de otros países de la región en los que las influencias fueron norteamericanas y de otros países europeos (Teixeira Soares, 1991).

También contextualizamos dicha apertura desde la perspectiva de género, ubicando algunas hipótesis en relación con el proceso de incorporación de las mujeres a la vida de la educación superior y universitaria de la época, en aspectos novedosos o emergentes (ej. profesionalización de las prácticas, remuneración de la tarea) y en otros que reeditaban los tradicionales roles sociales atribuidos a la mujer, como el cuidado de los otros, la atención de la salud, la subordinación a la figura masculina del médico, las damas rosadas y voluntarias (Nabergoi 2013a).

\section{Características de la propuesta formativa}

El plan de estudios de la carrera se estructuró con el fin de cumplir con los requisitos de la Federación Mundial de Terapeutas Ocupacionales (WFOT), creada pocos años antes. Ello supuso la incorporación de la formación en el campo de salud mental, que no formaba parte de las demandas de asistencia en el área física relacionadas con la polio, y la necesidad de realizar gestiones específicas para la aceptación local de dicha propuesta y la apertura del campo. Así, desde el comienzo el plan de estudios contempló la formación en los campos físico y mental con igual valoración. El currículo se estructuró con tres tipos de materias: asignaturas teóricas, actividades prácticas y práctica clínica, cuya distinción permanece en los currículos actuales. 
La ENTO se organizó con base en el modelo de escuela a la cual pertenecía mayoritariamente el equipo inglés contratado, la Dorset House School de Oxford, de la cual era directora la responsable del equipo. La Dorset House School fue la primera escuela de Terapia Ocupacional de Inglaterra, y se caracterizaba y diferenciaba de otras de la época, como la Escuela de Londres, por la selección estricta del curso de ingreso (estudiantes jóvenes con límite de edad superior de 35 años) y el énfasis en la necesidad de fundamentar científicamente la profesión, entre otras. Los aspectos formales así como los actitudinales eran muy enfatizados en la formación (Figura 2).

Figura 2. Manuscrito del plan de formación de la ENTO, 1959.

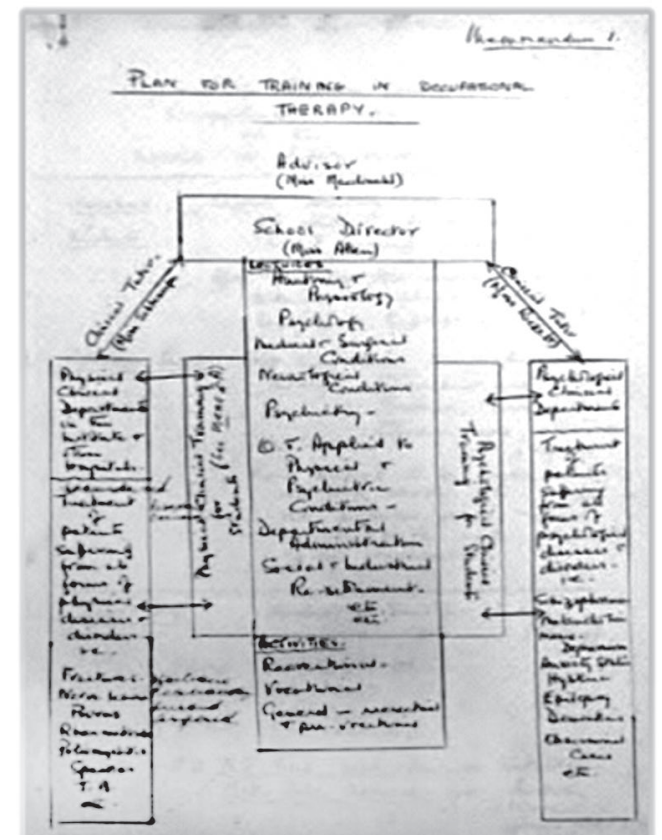

Manuscrito elaborado por Miss Mac Donald, directora de la Dorset House School y líder del equipo de terapistas inglesas contratadas por la CNRL para abrir la carrera en Argentina. Presenta un esquema de organigrama y estructura de Plan de Estudios con materias teóricas, actividades (centro) y prácticas clínicas en áreas física y psicológica (a los lados).

Foto: Archivo digital de las investigaciones llevadas a cabo por los autores.

En consecuencia, las primeras docentes inglesas eran muy jóvenes, aunque presentaban experiencia de trabajo en las áreas de su responsabilidad y conducían las materias específicas de la profesión. La responsable del equipo en calidad de asesora, Miss Macdonald $^{12}$, tenía al llegar a Argentina más de veinte años de experiencia en la dirección de la formación en su país y

\footnotetext{
${ }^{12}$ De esta forma la conocían y denominaban las entrevistadas, su nombre completo era Evelyn Mary Macdonald.
} 
había estado a cargo de la formación en Terapia Ocupacional en países de distintos continentes. De otra parte, los docentes de materias básicas eran locales, en su mayoría profesionales del Instituto de Rehabilitación, lo mismo ocurría con los docentes de actividades prácticas (Nabergoi, 2012, 2013a) (Figura 3).

Figura 3. Clase de telar en la ENTO, 1960 (probable).

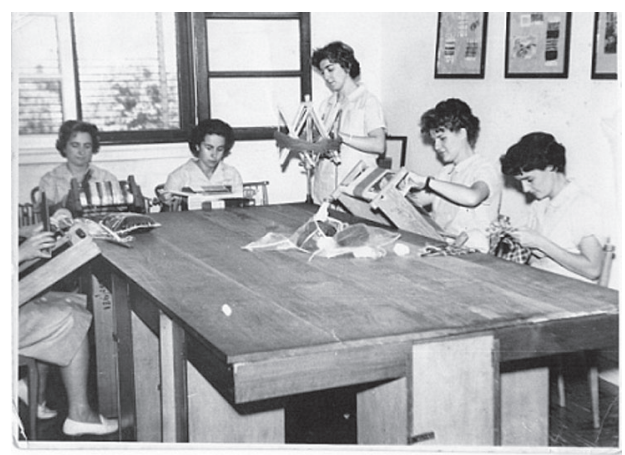

Foto: Contribución de Helvia Llambí.

Creación de nuevos espacios profesionales a través de programas de becas

La política pública nacional consistió en organizar, con instituciones de Capital Federal ${ }^{13}$ y las provincias, un sistema de becas a nivel federal a través de las cuales las estudiantes accedían a formarse en la ENTO y debían retornar a tales instituciones para abrir servicios de Terapia Ocupacional. Estas becas fueron otorgadas inicialmente con base en las recomendaciones de la terapista norteamericana Helen Dahlstrom y la fisioterapeuta Vivian Hannan. Entre 1961 y 1965 se recibieron en la ENTO 76 terapistas ocu- pacionales, más de la mitad de ellas provenían de 12 provincias del país y cinco llegaron a estudiar desde Chile. También estudiantes provenientes de otros países ingresaron a la ENTO por medio de becas y convenios nacionales e internacionales con organizaciones como la OPS/OMS, la Fundación Elizabeth Kenny y gobiernos de la región (OPS, 1994; Nabergoi et al, 2013a, 2013b).

De esta manera, en la ENTO se formaron profesionales que desarrollaron la disciplina en distintas regiones de Argentina y que, siendo argentinos o provenientes de la región, abrieron campos de actuación y escuelas en otros países de Latinoamérica, en el marco del desarrollo de una política regional en materia de rehabilitación (Bottinelli et al 2011b; Nabergoi et al 2013c).

\section{La formación para los campos físico y mental}

Uno de los requisitos de la propuesta formativa inicial fue que la misma preparara a los futuros terapistas ocupacionales para el desempeño tanto en el llamado campo físico como en el campo psiquiátrico o mental, con el argumento de que ello era necesario para el reconocimiento del título por la WFOT, creada apenas pocos años antes que la ENTO. De acuerdo con el Archivo Dorset House, esta titulación conjunta era el resultado de un acuerdo realizado en Inglaterra a partir de los límites que implicaba la formación especializada por campos. Este argumento, presente con fuerza en los

\footnotetext{
${ }^{13}$ Capital Federal es el distrito correspondiente a la actualmente denominada Ciudad Autónoma de Buenos Aires, capital de Argentina.
} 
discursos de las entrevistadas, fue una importante estrategia de validación de la propuesta formativa y fue utilizado para negociar el acceso a espacios de práctica clínica, el sostenimiento del contrato de las primeras docentes, entre otros, en diversas instancias institucionales y gubernamentales. El análisis de las transformaciones en el campo de salud mental a nivel local permitió reconocer que fueron estas las condiciones de posibilidad de la aceptación de un plan de estudios con tales características (Nabergoi, 2013b, 2013c; Bottinelli, Nabergoi, Mattei, Mullholland \& Spallato, 2013).

\section{Palabras finales}

Como producto de este trabajo y en el contexto del $50^{\circ}$ aniversario de la Terapia Ocupacional en Argentina (1959-2009), también realizamos la edición de un video documental homenaje titulado La ENTO. Un documental de la creación de la Escuela Nacional de Terapia Ocupacional en Argentina (Bottinelli et al, 2011a). Este fue realizado con base en las categorías y ejes de análisis que fueron surgiendo en el proceso de investigación. El material bibliográfico, documental y testimonial fue organizado y trabajado para su análisis y presentación en este material de 22 minutos. El cortometraje reúne material inédito, se destacan fotografías y videos de época facilitados por entrevistados y colaboradores, elementos de archivo de la Dorset House School y de la Biblioteca del ICRM-UNSAM, así como también extractos de entrevistas realizadas por el equipo de investigación.
El interés por la historia y el trabajo que hemos venido realizando también fue expresado por diferentes profesionales que hoy, insertos en servicios, refieren desconocer el origen y antecedentes de sus prácticas profesionales, dadas las vicisitudes de las políticas de salud, en particular en las áreas de rehabilitación y salud mental en nuestro país, que devinieron en suspensiones y momentos de reinicio de espacios de práctica, con escaso o nulo registro de las experiencias previas.

Es importante destacar también, que si bien la disciplina ha experimentado un crecimiento exponencial en la última década en Argentina, en la que se multiplicaron los espacios de formación y el número de alumnos y profesionales ${ }^{14}$, los mayores esfuerzos en el período reciente estuvieron destinados a la consolidación del nivel de grado. En este marco, la producción científico académica de la disciplina en nuestro país requiere aún de esfuerzos para impulsar su desarrollo en lo que refiere a la formación de recursos humanos en investigación, la incorporación de docentes y alumnos en la actividad científica, la promoción del aumento de la cantidad y la calidad de la producción y su puesta en circulación, que asegure la transferencia en los diferentes ámbitos y niveles de formación e intervención.

El proceso de profesionalización de la Terapia Ocupacional sufrió diferentes avatares desde la creación de la ENTO hasta la actualidad. Las líneas de indagación presentadas continuaron sien-

\footnotetext{
${ }^{14} \mathrm{Al}$ igual que en otras profesiones como Enfermería y Trabajo Social.
} 
do profundizadas por el equipo de investigación en los proyectos Creación e Institucionalización de la Terapia Ocupacional en Argentina. Los Comienzos de la Formación y el Desempeño Profesional de Terapeutas Ocupacionales en Argentina desde la Apertura de la Escuela Nacional de Terapia Ocupacional15 (2011-2012) y la tesis El Proceso de Transformación de la Atención Psiquiátrica hacia el Enfoque de Cuidados en Salud Mental en Argentina. Participación de Terapia Ocupacional en la Construcción del Campo de la Salud Mental en la Ciudad de Buenos Aires (1957-1976) (Nabergoi, 2013b). En este proceso hemos logrado diversos aprendizajes, hemos tenido la oportunidad de ver resurgir el interés por los inicios de la profesión en distintos países de Latinoamérica y de intercambiar experiencias con equipos de investigación de la región en el tema.

Actualmente la carrera de Licenciatura de Terapia Ocupacional en Argentina se dicta en 14 universidades nacionales. Algunos de los aspectos que marcaron los inicios de la formación profesional han permanecido hasta nuestros días, resignificados en los diversos contextos histórico sociales en los que tuvo su desarrollo, otros se han modificado significativamente. Dado que los contextos determinan la posibilidad de surgimiento, y que las perspectivas de los actores sociales involucrados permiten comprender el sentido de los mismos, realizar acciones que permitan historizar y comprender el surgimiento de un campo de conocimientos y prácticas,

\footnotetext{
${ }^{15}$ Proyectos SJ10/48 y R007 del ICRM-UNSaM.
}

así como su posicionamiento actual, contribuye a poder pensar en sus aportes y potencialidades a futuro, tanto al interior de la disciplina como respecto de la formación de profesionales y frente a las necesidades sociales que los convocan.

\section{Referencias}

Belmartino, S. (2005). La atención médica argentina en el siglo XX. Instituciones y procesos. Buenos Aires: S. XXI.

Belmartino, S., \& Bloch, C. (1994). El Sector Salud en la Argentina: Actores, Conflictos de intereses y modelos organizativos, 1960-1985. Buenos Aires: OPS. Nº40.

Bertaux, D. (1999). El enfoque biográfico: su validez metodológica, sus potencialidades. Proposiciones, 29, 1-23.

Bottinelli, M.M. (2003). Metodología de la investigación. Herramientas para un pensamiento complejo. Buenos Aires: Grafika Hels.

Bottinelli, M.M., Nabergoi, M., Pellegrini, M., Mulholland, M., Martínez del Pezzo Bredereke, M., Spallato, N., \& Zorzoli, J.M. (2011a). La ENTO. Un documental de la creación de la Escuela Nacional de Terapia Ocupacional. F.M. Díaz (Edición y realización) [Video documental]. Argentina: Universidad Nacional de San Martín.

Bottinelli, M.M., Nabergoi, M., Pellegrini, M., Mattei, M., Mulholland, M., Del Pezzo Bredereke, M., Spallato, N., \& Zorzoli, F. (2011b, octubre). Entramados internacionales en la historia de la formación de terapeutas ocupacionales. Indicios en los comienzos de la formación profesional en Argentina. En CLATO XII Congreso Brasilero de Terapia Ocupacional y IX Congreso Latinoamericano de Terapia Ocupacional. Sao Paulo, Brasil.

Bottinelli, M.M., Nabergoi, M., Mattei, M., Mulholland, M., \& Spallato, N. (2013, 
noviembre). El contexto de la educación superior en Argentina durante los inicios de la formación en terapia ocupacional. En Universidad de Buenos Aires, Memorias del V Congreso Internacional de Investigación y Práctica Profesional en Psicología. Buenos Aires, Argentina.

Collins, B. (1989). The Story of Dorset House School of Occupational Therapy 1930 1986. Oxford: Dorset House School of Occupational Therapy.

Daneri, S. (2005). Antecedentes de la terapia ocupacional en el Hospital José $T$. Borda. De la asunción del Dr. Domingo Cabred como director de la institución (1892) a la presencia efectiva del primer grupo de terapistas ocupacionales (1960) (Trabajo de integración final licencatura). Buenos Aires: Universidad Nacional de San Martín.

Daneri, S (mayo, 2010). Una mirada retrospectiva sobre el uso del trabajo y las actividades manuales, artísticas y recreativas en el tratamiento de la enfermedad mental en el Hospital José T. Borda. En WFOT, XV Congreso Mundial de Terapia Ocupacional: Ocupación desde Latinoamérica. Santiago de Chile, Chile.

Demiryi, M. (2001). La historia de Terapia Ocupacional en la Argentina: un análisis sociopolítico. En UNL y AETO, Libro de resúmenes de Terapia Ocupacional, 1er Encuentro del Litoral. Entre-Cruzadas y Paralelas (pp.116-117). Santa Fe, Argentina.

Destuet, S. (1999). Encuentros y marcas. Serie autores 1. Buenos Aires: COLTOA grupo editor.

Fernández, A., García, S., Duarte, C., \& Cruz, J. (2016, abril). Macroproyecto Historias de las Terapias Ocupacionales en Colombia. En L. Rodríguez, A. Fernández, S. García, C. Duarte, J. Cruz (Eds.), Historias de las Terapias Ocupacionales en Colombia, Boletín informativo Número 4.

Forn, C. (2007). Terapia ocupacional y rehabilitación psiquiátrica. Caracas: Edi- ciones del Vicerrectorado Académico UCV.

Fortain, M. (2003). Década del 50. Boletín de la Asociación Argentina de Terapistas Ocupacionales -AATO. Noviembre, 21-25.

Gracerón, M. (2000) Buscando otra mirada de la historia de la terapia ocupacional. Revista Materia Prima. 4 (15), 6-10.

Gómez, S. (2012). Antecedentes, creación y desarrollo de la terapia ocupacional en Chile: 50 años de historia. Santiago de Chile: Abarca Girard Ediciones.

Gomez Mengelberg, E. (2007a). Referentes históricos de la utilización de actividades en Sudamérica. En L. Paganizzi y otros. Terapia Ocupacional psicosocial. (p.p. 23-30).Buenos Aires: Ed Polemos.

Gómez Mengelberg, E. (2007b, noviembre,). Las condiciones socio-políticas de la creación de la carrera de Terapia Ocupacional en la República Argentina. En APSA, Actas del VIII Encuentro Argentino de Historia de la Psiquiatría, la Psicología y el Psicoanálisis. Mar del Plata, Argentina.

Guajardo, A. (2010, septiembre). Conferencia presentada en la Jornada Espacios Abiertos y Caminos Actuales en Derechos Humanos y Políticas en Terapia Ocupacional. Buenos Aires: Universidad Nacional de Quilmes..

Isuani, E. A. y Mercer, H. (1988). La fragmentación institucional del sector salud. Buenos Aires: Centro Editor de América Latina.

Kielhofner, G., \& Burke, J. P. (1977). Occupational therapy after 60 years: An account of changing identity and knowledge. American Journal of Occupational Therapy, 31, 675-689.

Da Rocha Medeiros, M.(2008). Terapia ocupacional. Un abordaje epistemológico y social. Santa Fe: Universidad Nacional del Litoral. 
De Souza Minayo, M.C.. (2009). La artesanía de la investigación cualitativa. Buenos Aires: Lugar Editorial.

Monzón, A., \& Risiga, M. (2004a, marzo). Espacio de reconstrucción de la memoria colectiva. Boletín de la Asociación Argentina de Terapistas Ocupacionales AATO. 9-15.

Monzón, A. \& Risiga, M. (2004b, junio). Espacio de reconstrucción de la memoria colectiva. Boletín de la Asociación Argentina de Terapistas Ocupacionales- AATO. 14-16,

Moruno, P. y Talavera, M.(comp.) (2007). Terapia Ocupacional. Una perspectiva histórica 90 años después de su creación. A Coruña: Asociación Profesional Gallega de Terapeutas Ocupacionales -APGTO. Disponible en: http://www.revistatog. com/mono/num1/mono1_esp.pdf

Nabergoi, M. (2012). Antecedentes de la ENTO. Sobre la creación de la Escuela de Terapia Ocupacional Dorset House en Inglaterra y la formación del equipo de terapistas inglesas que inició la carrera de Terapia Ocupacional en Argentina (Documento inédito).

Nabergoi, M. (2013a, junio). Caracterización de egresados de la Escuela Nacional de Terapia Ocupacional en el período 1961 - 1975. Aproximación a algunas cuestiones de género y clase. En Universidad Nacional de Quilmes, Segundas Jornadas de Iniciación en la Investigación Interdisciplinaria en Ciencias Sociales [CD]. Buenos Aires, Argentina.

Nabergoi, M. (2013b). El proceso de transformación de la atención psiquiátrica hacia el enfoque de cuidados en salud mental en Argentina. Participación de Terapia Ocupacional en la construcción del campo de la salud mental en la Ciudad de Buenos Aires (1957-1976). (Tesis de Doctorado en Salud Mental Comunitaria). Universidad Nacional de Lanús, Buenos Aires, Argentina.

Nabergoi, M. (2013c, mayo,). La transición del modelo inglés hacia una terapia ocu- pacional con identidad local: tres referentes históricos alternativos de terapia ocupacional en salud mental en los COmienzos de la profesión en Argentina. En XIII Jornadas de la Residencia de Terapia Ocupacional del Cobierno de la Ciudad de Buenos Aires [CD]. Buenos Aires, Argentina.

Nabergoi, M., Mattei, M., Bottinelli, M.M., Muholland, M., Martínez Del Pezzo Bredereke, M.M., Spallato, N., \& Zorzoli, F.J. (2011). Creación e institucionalización de la Terapia Ocupacional en Argentina. Los comienzos de la formación y el desempeño profesional de terapeutas ocupacionales en Argentina desde la apertura de la Escuela Nacional de Terapia Ocupacional. (Proyecto de investigación SJ10/48). Buenos Aires: Instituto de Ciencias de la Rehabilitación y el Movimiento, Universidad Nacional de San Martin.

Nabergoi, M., Mattei, M., Mulholland, M., Martínez Del Pezzo Bredereke, M., Spallato, N., Zorzoli, F., \& García Sartirana, A. (2013a, abril). Cooperación técnica en rehabilitación. Procesos iniciales de constitución de la formación académica y ejercicio profesional en Argentina. En Libros de resúmenes del $1^{\circ}$ Congreso Chileno y $8^{\circ}$ Jornadas Nacionales de Terapia Ocupacional. Viña del Mar, Chile.

Nabergoi, M., Mattei, M., García Sartirana, A., Briglia, J.,\& Zorzoli, F. (2013b, noviembre). Cooperación internacional y asistencia técnica en rehabilitación. Marcas y singularidades en los comienzos de la formación de terapeutas ocupacionales en Argentina. En Universidad de Buenos Aires, Memorias del V Congreso Internacional de Investigación y Práctica Profesional en Psicología. Buenos Aires, Argentina.

Nabergoi, M., Mattei, M., Martínez Del Pezzo Bredereke, M., Spallato, N., Zorzoli, F.,García Sartirana, A., \& Briglia J. (2013c, octubre). Los comienzos de la formación de Terapia Ocupacional en 
Argentina como parte de un entramado regional. Diálogos latinoamericanos. En $X$ Congreso Latinoamericano de Terapia Ocupacional y V Congreso Venezolano de Terapia Ocupacional Prácticas de la Terapia Ocupacional: Latinoamérica Construye e Integra Saberes. Caracas, Venezuela.

OPS (1994). Adelantos en medicina física y rehabilitación. Washington: OPS/OMS.

Paganizzi, L. (2007). Trabajo profesional de terapistas ocupacionales en la ciudad de Buenos Aires 1964-1996. En L. Paganizzi. Terapia Ocupacional Psicosocial (pp 31-58). Buenos Aires: Ed Polemos.

Peters, C.O. (2006). Power and professionalization in occupational therapy, 1950 until 1980 (Doctoral dissertation). New York University, New York.

Reed, K.L. (1998). Los comienzos de la Terapia Ocupacional. En H. Hopkins \& H. Smith, Williard \& Spackman Terapia Ocupacional. $8^{a}$ edición (pp.26-43). Madrid: Médica Panamericana.

Samaja, J. (2003). Epistemología y metodología, elementos para una teoría de la investigación científica. Colección Temas. Buenos Aires: Eudeba.

Sbriller, L. (1997). Introducción a Terapia Ocupacional. Marcos conceptuales. Buenos Aires: Ed. Catálogos.

Sbriller, L., \& Suter, M. (1985, 5 de septiembre). Desarrollo de Terapia Ocupacional en la República Argentina. En I Congreso Latinoamericano de Terapia Ocupacional. Porto Alegre, Brasil.

Schwartz, K. B. (2005) Historia de la Terapia Ocupacional. En Crepeau, Cohn \& Schell, Williard \& Spackman Terapia Ocupacional. 10 a edición (pp. 5-13). Buenos Aires: Ed Panamericana.

Spallato, N. (2010, mayo). Surgimiento de la primera Escuela Nacional de Terapia Ocupacional en Argentina: aspectos cronológicos. En WFOT, XV World Fede- ration of Occupational Therapists Congress. Santiago de Chile, Chile.

Teixeira Soares, L. B.(1991). Terapia Ocupacional, Lógica do Capital ou do Trabalho? Retrospectiva histórica da profissão no Estado brasileiro de 1950 a 1980. Sao Paulo: Huitec.

Testa, D. (2011). Poliomielitis: la "herencia maldita" y la esperanza de la rehabilitación. La epidemia de 1956 en la Ciudad de Buenos Aires. Intersitio. Revista Sociológica de Pensamiento Crítico, 5 (2). Disponible en http://www.intersticios.es/ article/view/8358

Testa, D. (2012). Aportes para el debate sobre los inicios de la profesionalización de la Terapia Ocupacional en Argentina. Revista Chilena de Terapia Ocupacional, 12 (1). Disponible en. http:// www.revistaterapiaocupacional.uchile. $\mathrm{Cl} /$ index.php/RTO/article/viewArticle/22054/23370

Testa, D.,\& Spampinato, S. (2010b). Género, salud mental y terapia ocupacional: algunas reflexiones sobre la influencia de la historia de las mujeres y la perspectiva de género en nuestras prácticas. Revista de Terapia Ocupacional de la Universidad de São Paulo, 21 (2), 174-181. Disponible en: http://www.revistas.usp.br/ rto/article/view/14101

Trujillo, A. (2002). Terapia Ocupacional, conocimiento y práctica en Colombia. Bogotá: Universidad Nacional de Colombia.

Valles, M.S. (2003). Técnicas cualitativas de investigación social. Reflexión metodológica y práctica profesional. Madrid: Ed. Síntesis.

Veronelli, J., \& Veronelli, M. (1991). Los orígenes institucionales de la Salud Pública en la Argentina. Buenos Aires: OPS/ OMS.

Villarroel, N. L. y equipo. (1985, 1 de julio). Campo ocupacional de los terapistas ocupacionales en la Argentina. En 1er 
Congreso Argentino de Terapia Ocupacional. La Rioja, Argentina.

Warschavski, G., \& Sbriller, L. (1990). Breve reseña histórica de terapia ocupacional. En G. Warschavski, \& L. Sbriller, Terapia Ocupacional. Teoría y Clínica. Buenos Aires: Ricardo Vergara Ediciones.

Wilcock, A.A. (2002). Occupation for health Volume 2: A Journey from prescription to self-health. London: British College of Occupational Therapists.
Zaldúa, G., Bottinelli, M., Lodieu, M., Perez Chavez, K., Pawlowicz, M., Gaillard, P., Tisera, A., \& Nabergoi, M. (2010). Salud Mental: territorios y narrativas entre la reproducción y la transformación. En G. Zaldúa.\& M. Bottinelli (coord.) Praxis psicosocial comunitaria en salud, pp 47-60. Buenos Aires: Eudeba. 